\title{
Three-Dimensional CFD Simulation of Fluid Flow inside a Vortex Tube on Basis of an Experimental Model- The Optimization of Vortex Chamber Radius
}

\author{
S. E. Rafiee* and M. M. Sadeghiazad \\ Department of Mechanical Engineering, Urmia University of Technology, Urmia, Iran \\ Email: seyed.ehsan.rafiee@gmail.com
}

\begin{abstract}
Vortex-chamber is a main part of vortex tube which the pressured gas is injected into this part tangentially. An appropriate design of vortex-chamber geometry leads to better efficiency and good vortex tube performance. In this study, the computational fluid dynamics (CFD) model is created on basis of an experimental model and is a three-dimensional (3D) steady compressible model that utilizes the k- $\varepsilon$ turbulent model. In this paper the effect of changing radius of vortex-chamber $\left(\mathrm{R}^{*}\right)$ on vortex tube performance has been studied for different value of $\mathrm{R}^{*}$ and the optimized radius of vortex-chamber has been determined. According to numerical results the cold temperature difference has increased when we take into account the effect of the radius of vortex-chamber in range of 5.7-11 mm and when the radius of vortex chamber has located in range of $11-13 \mathrm{~mm}$, the cold temperature difference has decreased. The highest $\Delta \mathrm{T}_{\mathrm{c}}$ is $46.77 \mathrm{~K}$ for $\mathrm{R}^{*}=11 \mathrm{~mm}$ at a cold mass fraction of 0.3 , higher than basic model around $6.3 \%$ at the same cold flow fraction. Finally, the results obtained, particularly the temperature values, are compared with some available experimental data, which show good agreement.
\end{abstract}

Keywords: Numerical simulation, Vortex tube, Vortex-chamber radius, Pressure drop, Cooling efficiency.

\section{INTRODUCTION}

The vortex tube is a device that has a simple geometry, without any moving or complicated parts that separates a pressurized gas into hot and cold streams. A schematic drawing of a typical vortex tube and its proceeds is shown in Fig. 1. A vortex tube includes different parts such as: one or more inlet nozzles, a vortex-chamber, a cold end orifice, a control valve that is located at hot end and finally a working tube. When pressured gas is injected into the vortex-chamber tangentially via the nozzle intakes, a strong rotational flow field is created. When the gas swirls to the center of the vortex-chamber it is expanded and cooled. After occurrence of the energy separation procedure in the vortex tube the pressured inlet gas stream was separated into two different gas streams including cold and hot exit gases. The "cold exit or cold orifice" is located at near the inlet nozzle and at the other side of the working tube there is a changeable stream restriction part namely the conical control valve which determines the mass flow rate of hot exit. As seen in Fig. 1, a percent of the compressed gas escapes through the conical valve at the end of the tube as hot stream and the remaining gas returns in an inner swirl flow and leaves through the cold exit orifice. Opening the hot control valve reduces the cold air flow and closing the hot valve increases the cold mass flow rate.

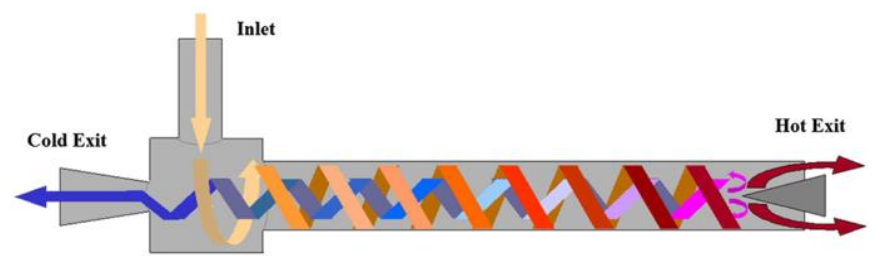

Figure 1. A schematic drawing of Ranque-Hilsch vortex tube

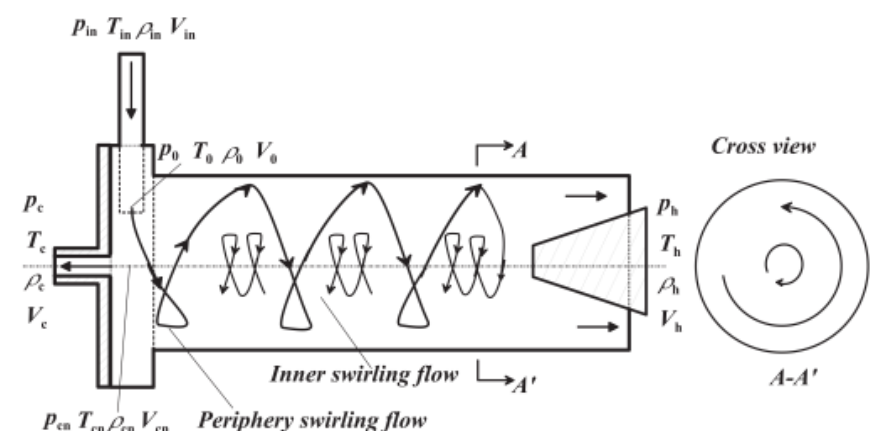

(a) 


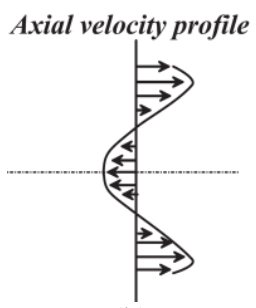

(b)

Figure 2. a) A schematic form of periphery and inner swirl flow inside the vortex tube. b) A schematic diagram of axial velocity profile inside the working tube

Cold mass fraction or $\alpha$ can been defined as below:

$\alpha=\frac{\dot{m}_{c}}{\dot{m}_{i}}$

In this equation $\dot{m}_{i}$ is the mass flow rate at the cold exit and $\dot{m}_{i}$ is the inlet flow rate.

The vortex tube air separator is discovered (for the first time) by a French researcher (Ranque in 1932 [1]). This device was geometrically improved by Rudolf Hilsch [2]. In this article we utilize the numerical models to explain the details of the separation process inside the air separator. Researches on the vortex tube air separators has a long history, however, we explain a brief list of important works as follow: The heat and mass transfer between the cold and hot cores (inside the vortex tube) is analyzed by Rafiee and sadeghiazad [3]. The capabilities of different turbulence models (the RSM, LES, $k-\omega, k-\varepsilon$ and $S S T k-\omega$ ) for predicting the flow structures within the air separator were examined by Baghdad et al. [4] and Rafiee and Sadeghiazad [5]. Some variations in the temperature drops are seen when a bended main tube is used in the structure of the air separator. These variations are reported in comparison with the air separator equipped with the straight main tube (Rafiee et al. [6], Valipour et al. [7]. Skye et al. [8] performed an experimental work on the thermal and flow separation inside a commercial vortex tube. The effect of divergent main tube has been investigated by Rahimi et.al [9] and the optimum angle for the divergent main tube has been achieved numerically. Some factors regarding the vortex tube structure (the inlet of slots, the ratio of slots, the hot and cold exit area, the rounding off edge radius, the internal radius of main tube and the convergent slots) were optimized by Rafiee and Sadeghiazad [10], Rafiee et al. [11], Pourmahmoud et al. [12] and Im et al. [13]. Some refrigerant gases (R728, R32, R134a, R161, $\mathrm{R} 744$, and R22) have been examined in the vortex tube air separator and the thermal performance of air separator has been studied and the best refrigerant gas has been determined (Pourmahmoud et al. [14] and Han et al. [15]). Rafiee and Sadeghiazad [16] analyzed the effect of different boundary conditions (pressure outlet and pressure far field) at the outlets and different working gases on the energy separation inside a vortex tube. Rafiee and Sadeghiazad [17 and 18] managed some experimental setups to optimize the control valve structural parameters such as the conical angle and the cone length and proved that there are some optimized values which lead to the best thermal capability. The convergent nozzles have been examined and optimized by Rafiee and Rahimi [19]. The impact of a new shape of the hot tube (the convergent main tube) is experimentally tested by Rafiee et al. [20]. Their results stated that there is an optimized angle for the convergent main tube to produce the best cooling capacity. Xue et al. [21] and Rafiee and Sadeghiazad [22] proposed a new energy explanation to analyze the thermal distribution and the exergy density inside the air separator applying the measured flow factors along the hot tube. The thermophysical parameters (the total temperature, the total pressure and the tangential velocity) inside the vortex tube are comprehensively reported by Rafiee and Rahimi [23]. A valuable work was done to analyze the isotope separation using vortex tubes by Lorenzini et al. [24]. The influence of inlet temperature on the vortex tube performance is investigated by Pourmahmoud et al [25]. In the presented work with assuming the advantages of using different radius of vortex chamber on the energy separation process and its considerable role on the creation of maximum cooling capacity of machine, the optimum radius is elected. This research believes that choosing an appropriate design of vortex-chamber is the one of important physical parameters for obtaining the highest refrigeration efficiency. So far numerical investigations towards optimization of vortex chamber radius has not been done but the importance of this object can be regarded as an interesting theme of research so that the machine would operate in the way that maximum cooling effect or maximum refrigeration capacity is provided.

\section{GOVERNING EQUATIONS}

The compressible turbulent and highly rotating flow inside the vortex tube is assumed to be three-dimensional, steady state and employs the standard k- $\varepsilon$ turbulence model on basis of finite volume method. The RNG k- $\varepsilon$ turbulence model and more advanced turbulence models such as the Reynolds stress equations were also investigated, but these models could not be converged for this simulation (Rafiee et al. [3]). Rafiee et al. [6] showed, for this reason that the numerical results has good agreement with the experimental data, the k- $\varepsilon$ model can be selected to simulate the effect of turbulence inside the computational domain. Consequently, the governing equations are arranged by the conservation of mass, momentum and energy equations, which are given by:

The equation for conservation of mass, or continuity equation, can be indicated as follows:

$\frac{\partial \rho}{\partial t}+\nabla \cdot(\rho \vec{v})=S_{m}$

The flow field in this investigation has been assumed 'steady state' and term $S_{m}$ is the mass added to continuous domain from other domains.

Momentum equation:

$\frac{\partial}{\partial \boldsymbol{X}_{j}}\left(\rho_{\boldsymbol{u}_{i}} \boldsymbol{u}_{j}\right)=-\frac{\partial p}{\partial \boldsymbol{X}_{i}}+\frac{\partial}{\partial \boldsymbol{X}_{j}}\left[\mu\left(\frac{\partial \boldsymbol{u}_{i}}{\partial \boldsymbol{X}_{j}}+\frac{\partial \boldsymbol{u}_{j}}{\partial \boldsymbol{X}_{i}}-\frac{2}{3} \delta_{i j} \frac{\partial \boldsymbol{u}_{k}}{\partial \boldsymbol{X}_{k}}\right)\right]+\frac{\partial}{\partial \boldsymbol{X}_{j}}\left(-\bar{\rho} \overline{\boldsymbol{u}}_{i}^{\prime} \overline{\boldsymbol{u}}_{j}^{\prime}\right)$

Energy equation:

$$
\begin{aligned}
& \frac{\partial}{\partial x_{i}}\left[u_{i} \rho\left(h+\frac{1}{2} u_{j} u_{j}\right)\right]=\frac{\partial}{\partial x_{j}}\left[k_{e f f} \frac{\partial T}{\partial x_{j}}+u_{i}\left(\tau_{i j}\right)_{e f f}\right], \\
& k_{e f f}=K+\frac{c_{p} \mu_{t}}{\operatorname{Pr}_{t}}
\end{aligned}
$$


Since we determined the working fluid is an ideal gas, then the compressibility effect must be considered as below:

$p=\rho R T$

The turbulence kinetic energy $(k)$ and the rate of dissipation $(\varepsilon)$ are obtained from the following equations:

$\frac{\partial}{\partial t}(\rho k)+\frac{\partial}{\partial x_{i}}\left(\rho k u_{i}\right)=\frac{\partial}{\partial x_{j}}\left[\left(\mu+\frac{\mu_{t}}{\sigma_{k}}\right) \frac{\partial k}{\partial x_{j}}\right]+G_{k}+G_{b}-\rho \varepsilon-Y_{M}$

$\frac{\partial}{\partial t}(\rho \varepsilon)+\frac{\partial}{\partial x_{i}}\left(\rho \varepsilon u_{i}\right)=\frac{\partial}{\partial x_{j}}\left[\left(\mu+\frac{\mu_{t}}{\sigma_{\varepsilon}}\right) \frac{\partial \varepsilon}{\partial x_{j}}\right]+C_{1 \varepsilon} \frac{\varepsilon}{k}\left(G_{k}+C_{3 \varepsilon} G_{b}\right)-C_{2 \varepsilon} \rho \frac{\varepsilon^{2}}{k}$

In these equations, $G_{k}, G_{b}$, and $Y_{M}$ represent the generation of turbulence kinetic energy due to the mean velocity gradients, the generation of turbulence kinetic energy due to buoyancy and the contribution of the fluctuating dilatation in compressible turbulence to the overall dissipation rate, respectively. $C_{1 \varepsilon}$ and $C_{2 \varepsilon}$ are constants. $\sigma_{k}$ and $\sigma_{\varepsilon}$ are the turbulent Prandtl numbers for $k$ and $\varepsilon$ also. The turbulent (or eddy) viscosity, $\mu_{t}$, is computed as follows:

$\mu_{t}=\rho C_{\mu} \frac{k^{2}}{\varepsilon}$

where, $C_{\mu}$ is a constant. The model constants $C_{1 \varepsilon}, C_{2 \varepsilon}, C_{\mu}, \sigma_{k}$ and $\sigma_{\varepsilon}$ have the following default values: $C_{1 \varepsilon}=1.44, C_{2 \varepsilon}=$ $1.92, C_{\mu}=0.09, \sigma_{k}=1.0, \sigma_{\varepsilon}=1.3$.

\section{PHYSICAL MODEL DESCRIPTION}

\subsection{D CFD model}

The 3D CFD model is created on basis of that was used by Skye et al. [7] in their experimental (Fig. 3) work. It is noteworthy that, an Exair ${ }^{\mathrm{TM}} 708$ slpm [16] vortex tube was used by Skye et al. [7] to perform all tests and to take all of the experimental data. The dimensional geometry of this vortex tube has been summarized in the Tab. 1. The 3D CFD mesh grid is shown in Fig. 4. In this model a regular organized mesh grid has been used. All radial line of this model of meshing has been connected to the centerline and the circuit lines have been designed organized from wall to centerline. So, the volume units that have been created in this model are regular cubic volumes. This meshing system helps the computations to be operated faster than the irregular and unorganized meshing, and the procedure of computations have been done more precisely.

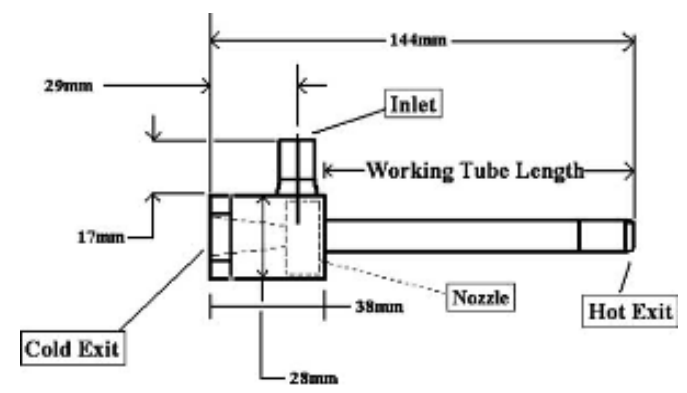

Figure 3. Schematic of vortex tube that used by Skye et al.
Table 1. Geometric measurements of the vortex tube that was used by Skye et al. [8]

\begin{tabular}{ll}
\hline Measurement & Value \\
\hline Working tube length & $106 \mathrm{~mm}$ \\
\hline Nozzle height & $0.97 \mathrm{~mm}$ \\
\hline Nozzle width & $1.41 \mathrm{~mm}$ \\
\hline Nozzle total inlet area (An) & $8.2 \mathrm{~mm}^{2}$ \\
\hline Cold exit diameter & $6.2 \mathrm{~mm}$ \\
\hline Cold exit area & $30.3 \mathrm{~mm}^{2}$ \\
\hline Hot exit diameter & $11 \mathrm{~mm}$ \\
\hline Hot exit area & $95 \mathrm{~mm}^{2}$ \\
\hline
\end{tabular}

For this reason the CFD model has been assumed a rotational periodic condition. Hence, only a sector of the flow domain with angle of $60^{\circ}$ needs to consider for computations as shown in Fig. 3.

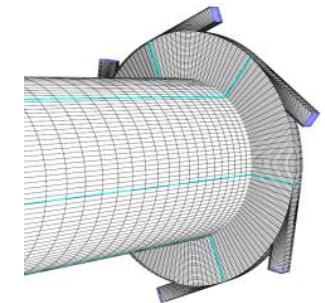

(a)

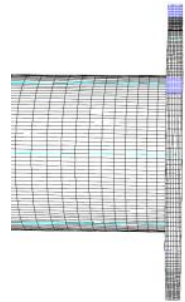

(b)

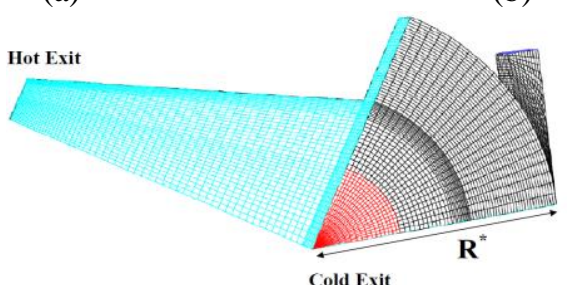

(c)

Figure 4. a) and b) 3D CFD model of vortex chamber with six straight nozzles. c) A sector of computational domain

\subsection{Boundary conditions}

Boundary conditions for this study have been indicated in Fig. 4. The inlet is modeled as mass-flow-inlet .The inlet stagnation temperature and the total mass flow inlet are fixed to $294.2 \mathrm{~K}$ and $8.35 \mathrm{~g} \mathrm{~s}^{-1}$ respectively according to experimental conditions. A no-slip boundary condition is used on all walls of the system. For the cold and hot exits, two kinds of boundary condition can be used for numerical analysis. The first boundary condition is pressure-outlet and the second one can be considered as pressure-far-field. The pressure-outlet boundary condition is used when the outlet pressures on both cold and hot outlets are known. However, the pressure-far-field boundary condition is used for the models with unknown outlet pressures.

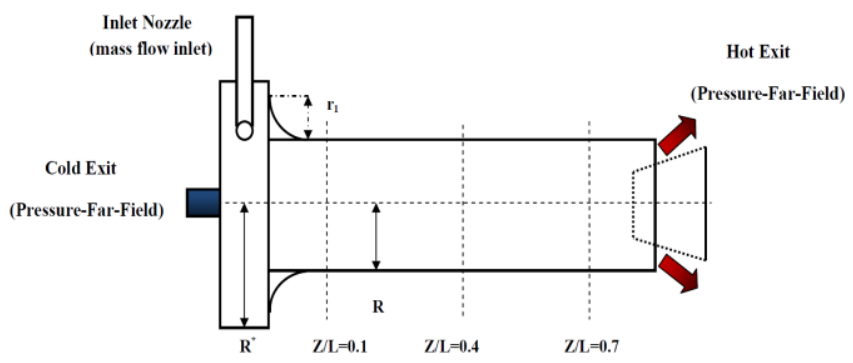

Figure 5. A schematic form of boundary conditions 
In this article both kinds of these boundary conditions are used for cold and hot exits. For pressure-outlet boundary condition, the static pressure at the cold exit boundary was fixed at experimental measurements pressure and the static pressure at the hot exit boundary is adjusted in the way to vary the cold mass fraction. The pressure-far-field boundary condition which is also employed for both exhausts including hot and cold exit is a state when the static pressures at the exhausts of vortex tube are not determined exactly. In the other hand, a vortex tube usually works in the ambient condition and for change in the cold mass fraction one need to change the area of hot exit that is the true action for this purpose in comparison with pressure-outlet boundary condition. In this article, both kinds of boundary conditions are applied and investigated numerically and the results compare to each other. The computations in this study utilize a pressure correction based iterative SIMPLE algorithm for discretising the convective transport terms. A compressible form of the Navier-Stokes equation along with the standard k$\varepsilon$ model by second order upwind for momentum, turbulence and energy equations has been used to simulate the phenomenon of flow pattern and temperature separation in a vortex tube with 6 straight inlet nozzles operating under condition of using different geometry of vortex-chamber by using the FLUENT ${ }^{\mathrm{TM}}$ software package. The default values of under-relaxation factor are shown in Table 2.

Table 2. Under-relaxation factors used for computations

\begin{tabular}{ll}
\hline Under-Relaxation Factor & Value \\
\hline Pressure & 0.3 \\
\hline Density & 1 \\
\hline Body Force & 1 \\
\hline Momentum & 0.7 \\
\hline Turbulent Kinetic Energy & 0.8 \\
\hline Turbulent Dissipation Rate & 0.8 \\
\hline Turbulent Viscosity & 1 \\
\hline Energy & 1 \\
\hline
\end{tabular}

\section{RESULTS AND DISCUSSION}

The numerical results obtained from the models, which involve the effect of vortex-chamber radius change on different vortex tube thermo and physical characteristics, are presented in this section.

\subsection{Validation}

The previous CFD researches on vortex tube usually have been performed in the fixed boundary condition such as pressure at the hot and cold exits. These values of pressure at exhausts are taken from experimental process that has been achieved during the experiment procedure. Since these pressures are not available always, this paper utilizes a useful method with any necessity of pressure values at cold and hot exit. This method is useful and suitable for CFD researchers that are involved in vortex tube fields. In this method, the pressure values of exhausts in the vortex tube are not necessary to be available and the prediction is independent of pressure values at the exhausts. In this paper and this method the pressure boundary condition adjusted as pressure-far-field condition. This means that the vortex tube is working in ambient conditions. In order to achieve the certain cold mass fraction, we have to change the area of hot exhaust. In the real state (Experimental) changing the hot exit area is achieved by the action of hot control valve. In this section, the both kinds of boundary conditions for exhaust i.e. pressure-far-field boundary condition and pressure-outlet boundary condition are applied to the CFD model together with other boundary conditions for inlet, wall and periodic planes which described in section 3.2 and cold and hot exit temperatures are derived and compared with experimental data to demonstrate that there is not noticeable difference for these two kinds of boundary conditions. As seen in Fig. 5 and 6, cold and hot exit temperatures for both boundary conditions as a function of cold mass fraction $(\alpha)$ have good agreement with the experimental data. The presented data in Fig. 5 and 6 are the minimum and maximum temperature achieved for cold and hot exits respectively. In Fig. 5 the minimum $\mathrm{T}_{\mathrm{c}}=250.24 \mathrm{~K}$ and $\mathrm{T}_{\mathrm{c}}=249.6 \mathrm{~K}$ is obtained at about $\alpha=0.3$ through the $\mathrm{CFD}$ simulations and experiments respectively. The maximum and minimum difference between CFD results and experimental data in cold temperatures is about $3.32 \%$ and $0.18 \%$ respectively. This difference for hot temperature at the peak of its value reaches to $3.77 \%$. As seen in Fig. 6, the applied 3D CFD model can produce maximum hot gas temperature of $354.34 \mathrm{~K}$ at $\alpha=0.86$ and a minimum cold gas temperature of $250.24 \mathrm{~K}$ at about 0.3 cold mass fraction. It should be mentioned that the validation part has been done for compressed air as operation fluid.

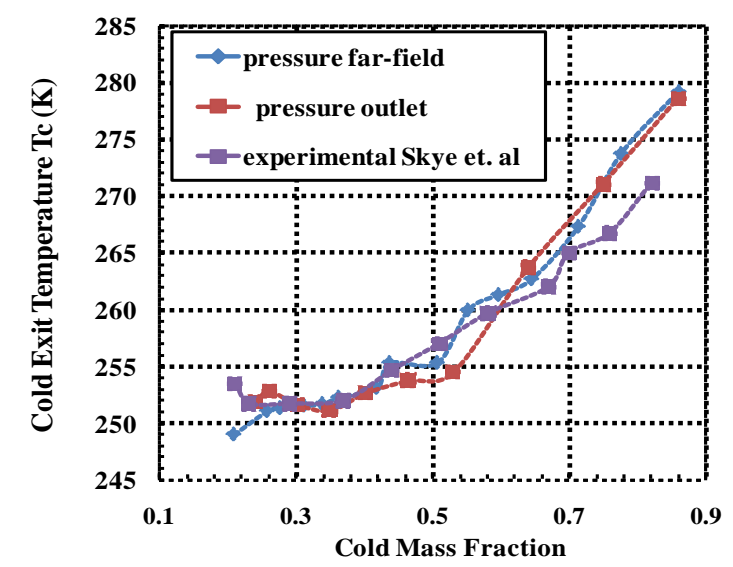

Figure 6. Comparison of cold exit temperature for both kinds of exhausts boundary conditions with the experimental data

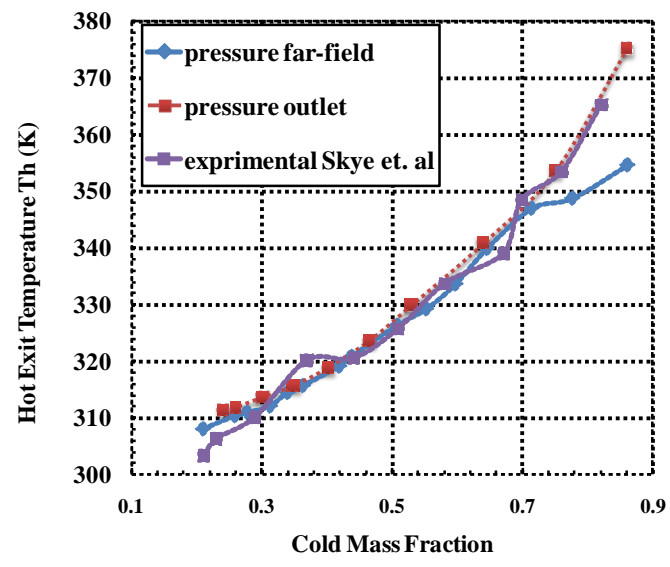

Figure 7. Comparison of hot exit temperature for both kinds of exhausts boundary conditions with the experimental data 


\subsection{Grid independence study}

The 3D CFD analysis has been performed for different average unit cell volumes in vortex tube as a computational domain. This is for the reason that removing probable errors arising due to grid coarseness. Therefore, first the grid independence study has been done for $\alpha=0.3$. As seen in the Fig. 6, at this cold mass fraction the vortex tube (with 6 straight nozzles) achieves a minimum outlet cold gas temperature. Consequently, in the most of the evaluations we use $\alpha=0.3$ as a special value of cold mass fraction.

The variation of cold exit temperature difference and maximum tangential velocity as the main parameters are shown in Figs. 8 and 9 respectively for different unit cell volumes. Not much major advantage can be seen in reducing of the unit cell volume size below $0.026 \mathrm{~mm}^{3}$; which corresponds to 287000 cells.

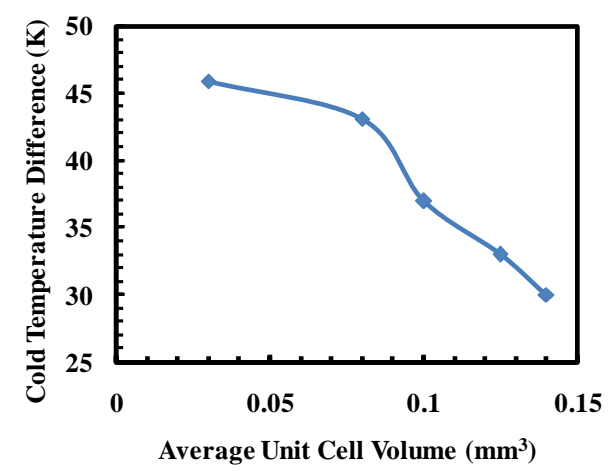

Figure 8. Grid size independence study on cold temperature difference at different average unit cell volume

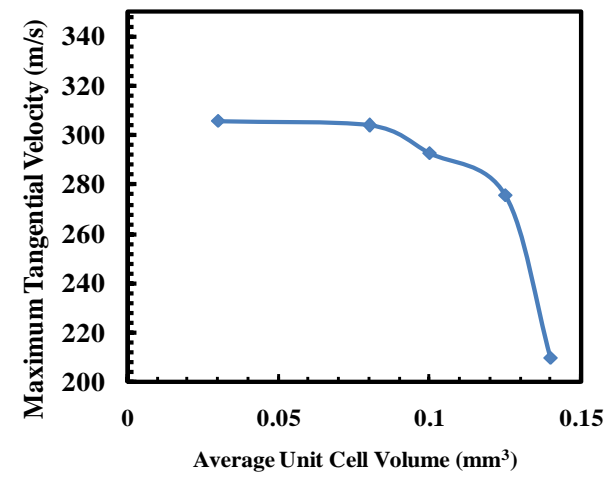

Figure 9. Grid size independence study on maximum swirl velocity at different average unit cell volume

\subsection{An investigation into the optimization of $R^{*}$}

In this section the effect of changing radius of vortexchamber $\left(\mathrm{R}^{*}\right)$ on vortex tube performance has been studied for seven different values $\left(\mathrm{R}^{*}=5.7,6.4,7,9,11,12\right.$ and 13) of $\mathrm{R}^{*}$. For each of these models, a 3D CFD model has been created and the results of computations in these models have been compared with each other. The compared results are: tangential velocity, total temperature and total pressure. The total temperature separation results for the seven cases are provided in Tab. 3, below:
Table 3. Total temperature separation at cold mass fraction $\alpha=0.3$ in the CFD models

\begin{tabular}{llccccc}
\hline Model & $\begin{array}{l}\mathrm{R}^{*} \\
(\mathrm{~mm})\end{array}$ & $\mathrm{T}_{\mathrm{c}}(\mathrm{K})$ & $\mathrm{T}_{\mathrm{h}}(\mathrm{K})$ & $\Delta T_{c}(\mathrm{~K})$ & $\begin{array}{l}\Delta T_{h} \\
(\mathrm{~K})\end{array}$ & $\begin{array}{l}\Delta T_{t} \\
(\mathrm{~K})\end{array}$ \\
\hline Case (a) & 5.7 & 250.2 & 311.5 & 43.96 & 17.3 & 61.26 \\
\hline Case (b) & 6.4 & 249.3 & 311.7 & 44.81 & 17.5 & 62.36 \\
\hline Case (c) & 7 & 249.3 & 312.7 & 44.9 & 18.5 & 63.47 \\
\hline Case (e) & 9 & 247.6 & 313.4 & 46.55 & 19.2 & 65.8 \\
\hline Case (g) & 11 & 247.4 & 313.3 & 46.77 & 19.1 & 65.95 \\
\hline Case (h) & 12 & 248.1 & 313.7 & 46.1 & 19.5 & 65.61 \\
\hline Case (i) & 13 & 248.4 & 313.5 & 45.77 & 19.3 & 65.16 \\
\hline
\end{tabular}

As Table 3, this is clearly observable that the cold temperature difference magnitude in the model of $\mathrm{R}^{*}=11 \mathrm{~mm}$ [Case $(\mathrm{g})]$ is the greatest value among the all of created models.

\subsubsection{Swirl (Tangential) velocity}

In order to demonstrate the effect of change in radius of vortex-chamber on radial profiles of tangential velocity, the profiles at two axial locations $(\mathrm{Z} / \mathrm{L}=0.1$ and 0.7$)$ at the cold mass fraction 0.3 were analyzed, the diagrams of which are depicted in Fig. 10 and Fig.11.

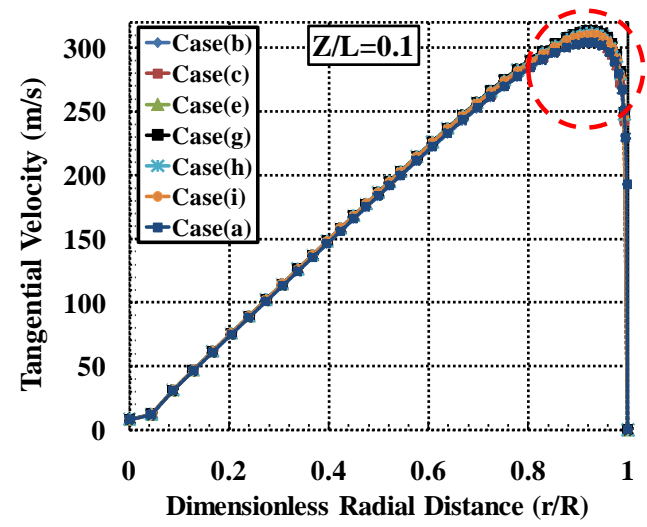

(a)

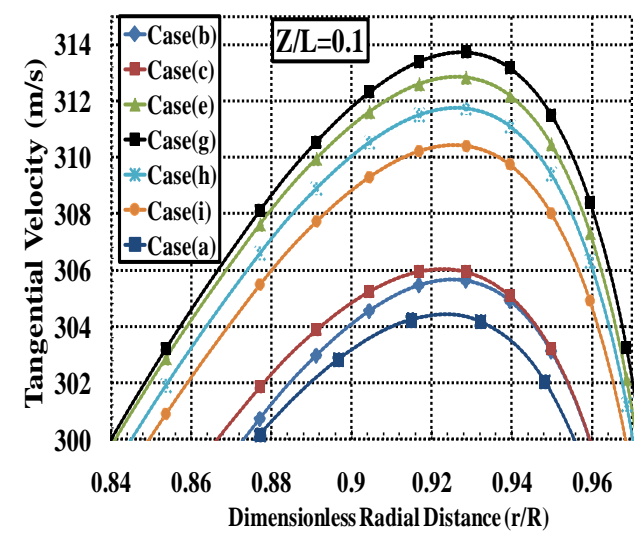

(b)

Figure 10. a) Radial profile of tangential velocity at $\mathrm{Z} / \mathrm{L}=0.1$ for $\alpha=0.3$. b) The marked area on Fig.10.a

The diagrams show that at $\mathrm{Z} / \mathrm{L}=0.1$ (near the chamber), the maximum magnitude of tangential velocity in the model of $\mathrm{R}^{*}=11 \mathrm{~mm}$ [Case $\left.(\mathrm{g})\right]$ is $313.8 \mathrm{~m} / \mathrm{s}$ as the greatest value of all other models, whereas this value for basic model is $304.2 \mathrm{~m} / \mathrm{s}$. This means that the optimization of radius of vortex-chamber leads to increase in maximum swirl velocity about $3 \%$ at near 
of cold exhaust. In Fig. 10 and 11, the radial profiles for the tangential velocity at $\mathrm{Z} / \mathrm{L}=0.1$ and 0.7 are provided for all of the created models. The swirl (tangential) velocity magnitude decreases as we move towards the hot end exit. This is clearly observable that the tangential velocity magnitude in the model of $\mathrm{R}^{*}=11 \mathrm{~mm}$ [Case $(\mathrm{g})$ ] is the greatest swirl velocity among the all of created models and the tangential velocity drop magnitude in the model of $\mathrm{R}^{*}=9,11$ and $12 \mathrm{~mm}$ is $119.2 \mathrm{~m} / \mathrm{s}$, $120.6 \mathrm{~m} / \mathrm{s}$ and $120.3 \mathrm{~m} / \mathrm{s}$ respectively as the greatest swirl velocities drop among the all of created models. This velocity drop is the sign for appropriate energy separation procedure inside the vortex tube. The radial profile of the tangential velocity shows a free vortex near the wall. On the other hand, another, forced, vortex is formed in the core, in which swirl velocity values decrease as we move towards the centerline of working tube. The results of Fig 10 and 11 are in good coordination with the observations of Kurosaka [4], Gutsol [17]. The upper Figures are drawn at two section of working tube and describe the tangential velocity pattern inside the working tube whereas the swirl velocity inside the vortexchamber has different pattern. Figure 12 shows the variation of swirl velocity inside the vortex-chamber and working tube.

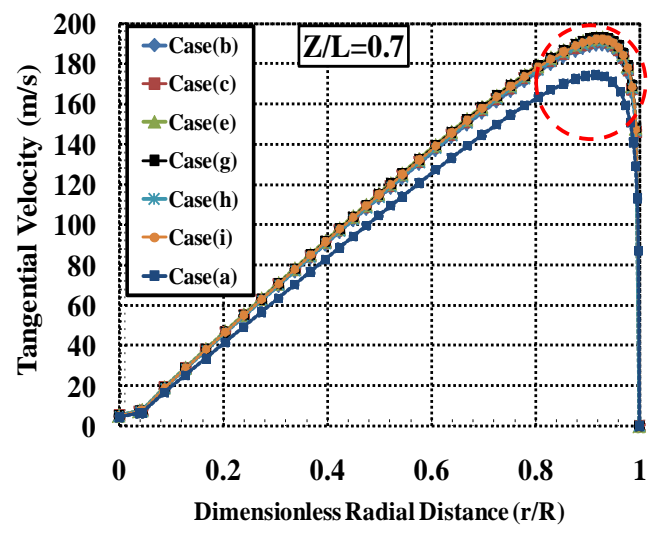

(a)

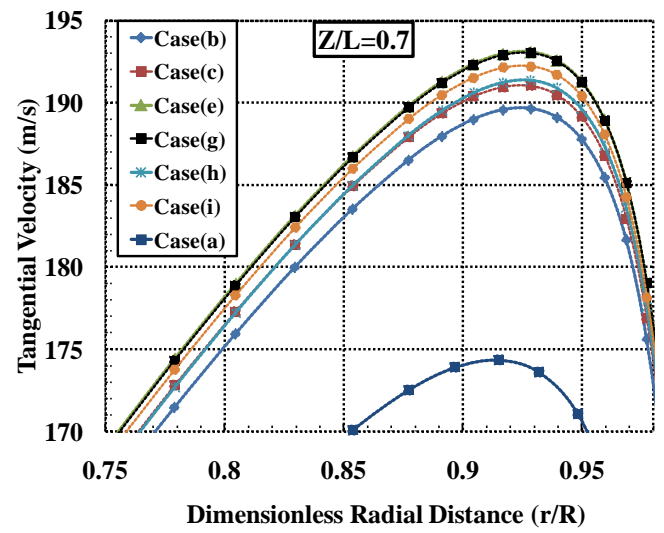

(b)

Figure 11. a) Radial profile of tangential velocity at $\mathrm{Z} / \mathrm{L}=0.7$ for $\alpha=0.3$. b) The marked area on Fig.11.a

where, $R_{V C}, R_{V T}$ and $R_{C}$ are the radiuses of vortex-chamber, vortex tube and cold orifice respectively.
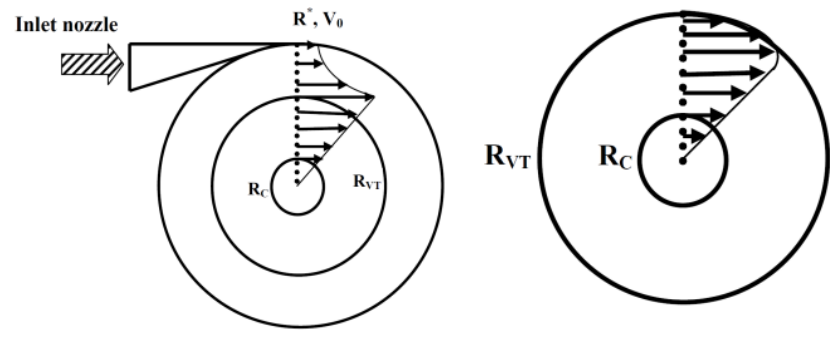

Figure 12. a) Velocity distribution in the vortex-chamber. b) Velocity pattern in the working tube

\subsubsection{Total temperature and total pressure}

The total temperature variations for different radius of vortex-chamber at $\mathrm{Z} / \mathrm{L}=0.1$ (near the cold exit) are presented in Fig 13.

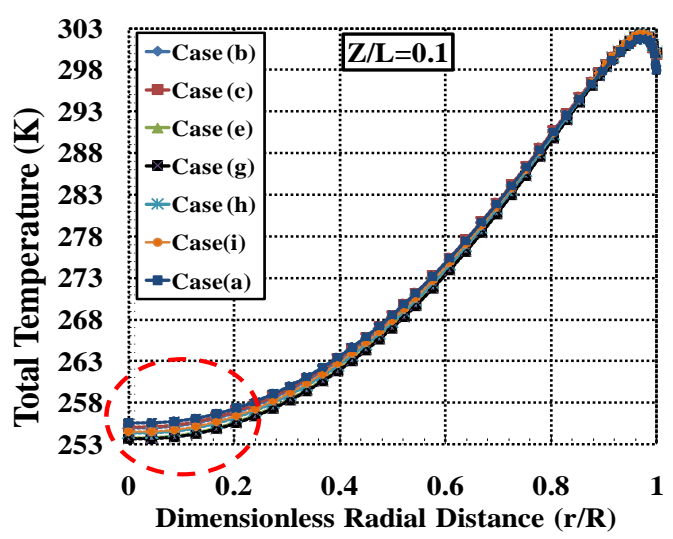

(a)

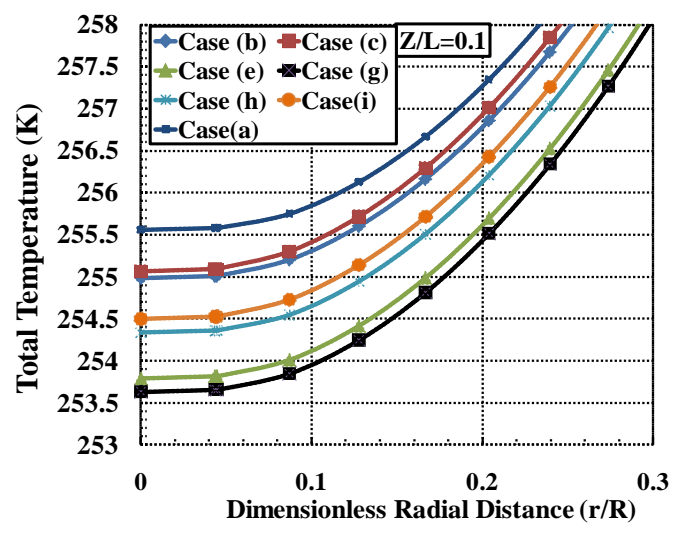

(b)

Figure 13. a) Radial profile of total temperature at $\mathrm{Z} / \mathrm{L}=0.1$ for $\alpha=0.3$. b) The marked area on Fig.13.a

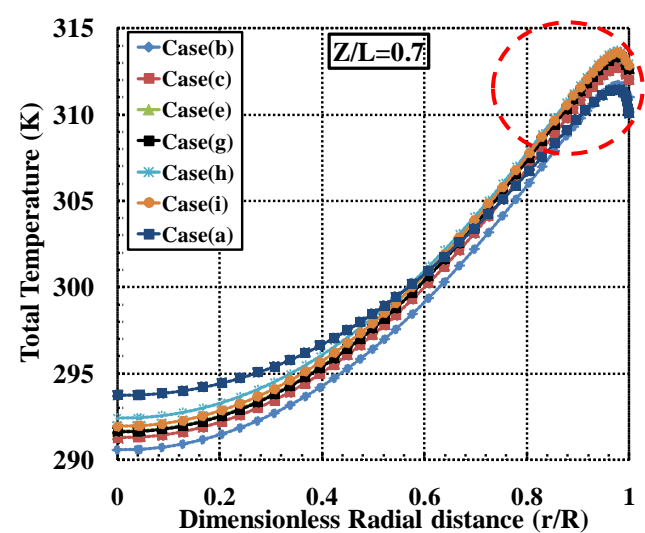

(a) 


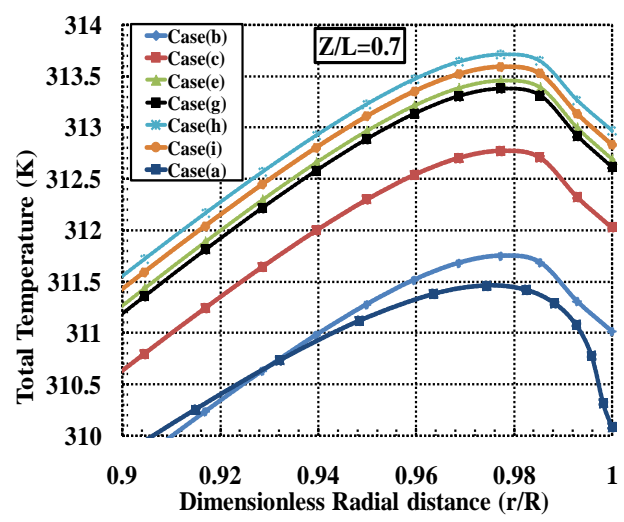

(b)

Figure 14. a) Radial profile of total temperature at $\mathrm{Z} / \mathrm{L}=0.7$ for $\alpha=0.3$. b) The marked area on Fig.14.a

As seen in Fig. 13, the minimum total temperature near the cold exit $(\mathrm{Z} / \mathrm{L}=0.1)$ is $253.6 \mathrm{~K}$ and is occurred by case $(\mathrm{g})$, whereas this value for basic model is $255.57 \mathrm{~K}$. This means that the optimization of radius of vortex-chamber leads to decrease in minimum cold temperature about $2 \mathrm{~K}$ at near of cold exhaust. As shown in Fig. 14, the maximum total temperature near the hot exit $(\mathrm{Z} / \mathrm{L}=0.7)$ is obtained by case (h). For all of $\mathrm{R}^{*}$ values, the maximum total temperature achieved near the periphery of the tube wall. The low temperature zone in the core coincides with the negligible tangential velocity zone. Figures 15 and 16 show the total pressure variations for different radius of vortex-chamber at the two axial locations $\mathrm{Z} / \mathrm{L}=0.1$ and 0.7 as a function of dimensionless radial distance of working tube. The maximal magnitude of total pressure is observed near the periphery of the tube wall. The diagrams show that at $\mathrm{Z} / \mathrm{L}=0.1$ (near the chamber), the maximum magnitude of total pressure in the model of $\mathrm{R}^{*}=11 \mathrm{~mm}$ [Case $\left.(\mathrm{g})\right]$ is $374.1 \mathrm{kpa}$ as the greatest value of all other models, whereas this value for basic model is $339.24 \mathrm{kpa}$. So the optimization of radius of vortexchamber creates an improvement in maximum total pressure about $10.32 \%$ at near of cold exit. These results reveal that the temperature reduction of cold air, the total pressure value and the tangential velocity value of fluid stream are substantially influenced by the radius of vortex-chamber.

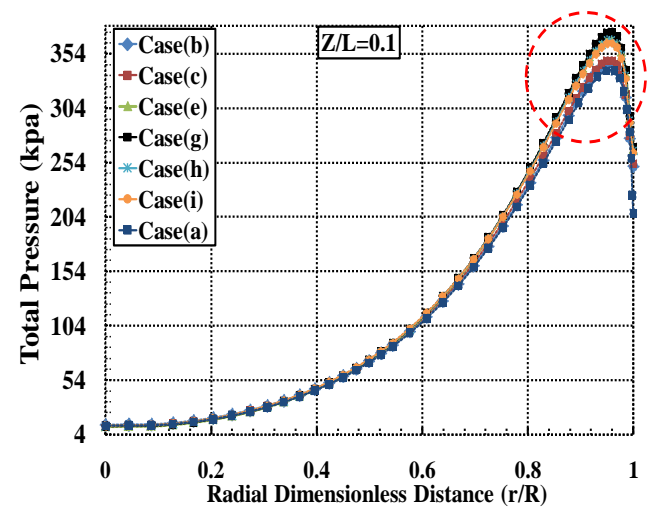

(a)

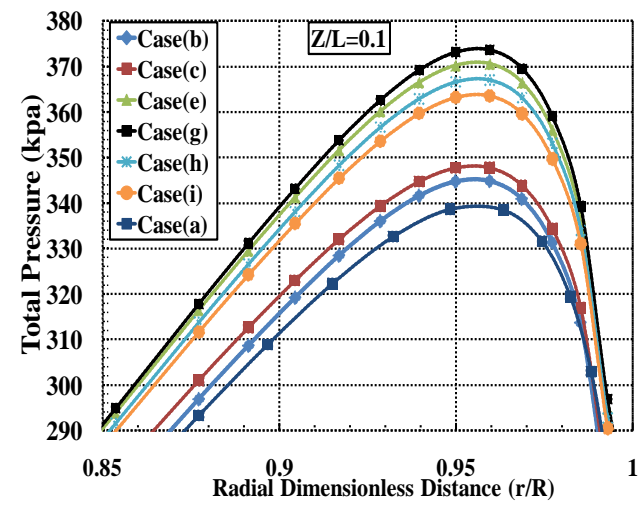

(b)

Figure 15. a) Radial profile of total pressure at $\mathrm{Z} / \mathrm{L}=0.1$ for $\alpha$ $=0.3$. b) The marked area on Fig.15.a

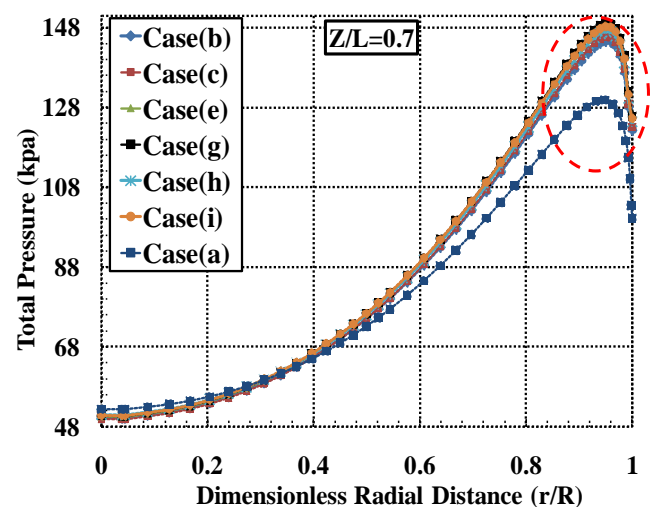

(a)

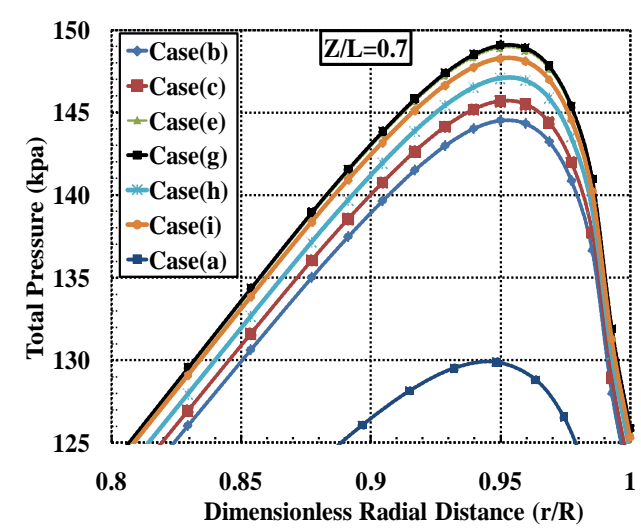

(b)

Figure 16. a) Radial profile of total pressure at $\mathrm{Z} / \mathrm{L}=0.7$ for $\alpha$ $=0.3$. b) The marked area on Fig.16.a

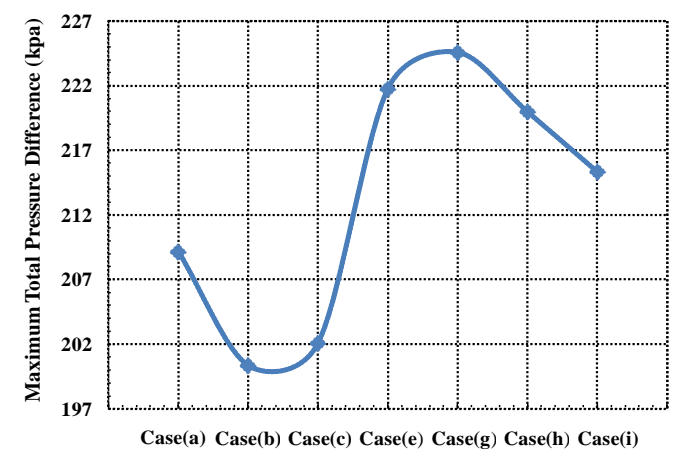

Figure 17. Maximum total pressure drop between $\mathrm{Z} / \mathrm{L}=0.1$ and $\mathrm{Z} / \mathrm{L}=0.7$ 
As seen in Fig. 15 and 16, fluid stream has greater total pressure in case $(\mathrm{g})$ in comparison with other cases. Also maximum total pressure difference between $\mathrm{Z} / \mathrm{L}=0.1$ and $\mathrm{Z} / \mathrm{L}=0.7$ can be observed in Fig. 17. According to this Figure, case $(\mathrm{g})$ has the greatest value of pressure drop across the working tube among the all of created models. Figures 13 to 16 demonstrate that the case $(\mathrm{g})$ has better total temperature, total pressure and the efficient total temperature than other cases. Case (g) has lower cold exit temperature than the other models. The most important purpose of this study and optimization is the increasing total pressure and tangential velocity at the junction of vortex-chamber and working tube also the maximum pressure drop and velocity drop across the working tube which is shown in the pressure and velocity Figures. These conditions is the most important sign of appropriate energy separation procedure inside the vortex tube which leads to increase of cold temperature difference and better cooling capacity. The contours of total temperature of optimum model for the inlet gas temperature $294.2 \mathrm{~K}$ and cold mass fraction 0.3 are shown in Fig. 18.

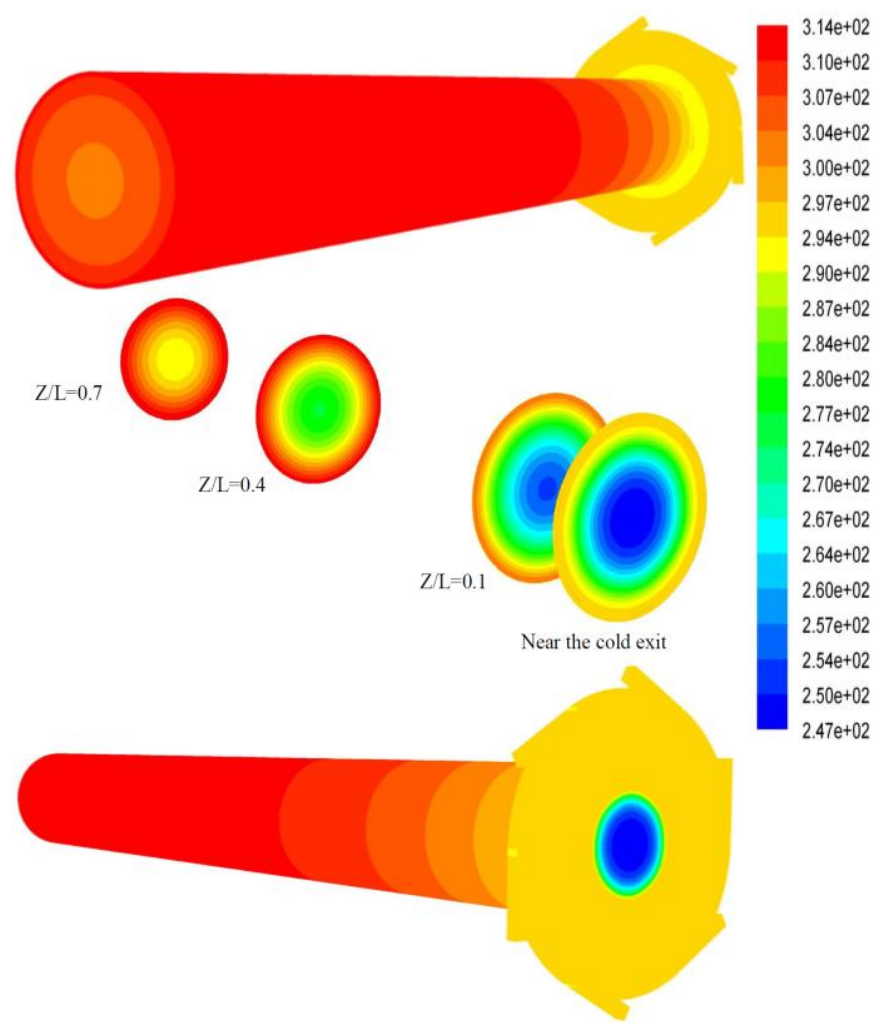

Figure 13. Contours of total temperature of the optimum model at $\mathrm{Ti}=294.2 \mathrm{~K}$.

\section{CONCLUSIONS}

This paper presents the results of a series of numerical simulations focusing on various geometries of the "vortexchamber" for constant inlet mass flow rate and cold mass fraction. Specifically, the models were conducted using different vortex-chamber diameters radius at tube's entrance inside the vortex-chamber. The most important purpose of this study and optimization is the increasing total pressure and tangential velocity at the junction of vortex-chamber and working tube furthermore creation of the maximum pressure drop and velocity drop across the working tube which is shown in the pressure and velocity Figures. These conditions is the most important sign of appropriate energy separation procedure inside the vortex tube which leads to improvement of cold temperature difference and better cooling capacity. In this investigation, the numerical results have been obtained for a counter flow Ranque-Hilsch vortex tube having $\mathrm{R}^{*}=5.7$, $6.4,7,9,11,12,13 \mathrm{~mm}$; L/D=9.298 when used with air as system fluid. Cold mass fraction was fixed at $\alpha=0.3$. The results present that there is the optimum value of $\mathrm{R}^{*}$ for obtaining the highest refrigeration efficiency, and $11 \mathrm{~mm}$ vortex-chamber radius is the optimal candidates under our computational conditions. According to numerical results the total temperature difference has increased when we take into account the effect of the radius of vortex-chamber in range of $5.7-11 \mathrm{~mm}$ and when the radius of vortex-chamber has located in range of $11-13 \mathrm{~mm}$, the total temperature difference has decreased. It has been seen that the maximum total temperature difference is achieved when $\mathrm{R}^{*}=11 \mathrm{~mm}$ [case $\left.(\mathrm{g})\right]$ among the seven primitive CFD models. The highest $\Delta T_{c}$ is $46.77 \mathrm{~K}$ for $\mathrm{R}^{*}=11 \mathrm{~mm}$ at a cold mass fraction of 0.3 , higher than basic model around $6.3 \%$ at the same cold flow fraction.

\section{REFERENCES}

[1] G. J. Ranque, "Experiments on expansion in a vortex with simultaneous exhaust of hot air and cold air," $L e$ Journal de Physique et le Radium, vol. 4, pp. 112-114, 1933.

[2] R. Hilsch, "The use of expansion of gases in a centrifugal field as a cooling process," Rev. Sci. Instrum, vol. 18, pp. 108-113, 1947. DOI: 10.1063/1.1740893.

[3] S. E. Rafiee and M. M. Sadeghiazad, "Heat and mass transfer between cold and hot vortex cores inside ranque-hilsch vortex tube-optimization of hot tube length," International Journal of Heat and Technology, Vol. 34(1), pp. 31-38, 2016. DOI: 10.18280/ijht.340105.

[4] M. Baghdad, A. Ouadha, O. Imine and Y. Addad, "Numerical study of energy separation in a vortex tube with different RANS models," Int. J. Thermal Sciences, vol. 50, no. 12, pp. 2377-2385, 2011. DOI: 10.1016/j.ijthermalsci.2011.07.011.

[5] S. E. Rafiee and M. M. Sadeghiazad, "Threedimensional computational prediction of vortex separation phenomenon inside Ranque-Hilsch vortex tube," Aviation, vol. 20, no. 1, 2016, pp. 21-31 DOI: 10.3846/16487788.2016.1139814.

[6] S. E. Rafiee, S. Ayenehpour and M. M. Sadeghiazad, "A study on the optimization of the angle of curvature for a Ranque-Hilsch vortex tube, using both experimental and full Reynolds stress turbulence numerical modelling." Heat and Mass Transfer, February 2016, vol. 52, no. 2, pp. 337-350, 2016. DOI: $10.1007 / \mathrm{s} 00231-015-1562-\mathrm{y}$.

[7] M. S. Valipour and N. Niazi, "Experimental modeling of a curved Ranque-Hilsch vortex tube refrigerator," Int. J. refrigeration, vol. 34, pp. 1109-1116, 2011. DOI: 10.1016/j.ijrefrig.2011.02.013.

[8] H. M. Skye, G. F. Nellis and S. A. Klein, "Comparison of CFD analysis to empirical data in a commercial vortex tube," Int. J. Refrig, vol. 29, pp. 7180, 2006. DOI:10.1016/j.ijrefrig.2005.05.004

[9] M. Rahimi, S. E. Rafiee and N. Pourmahmoud, "Numerical investigation of the effect of divergent hot tube on the energy separation in a vortex tube," 
International Journal of Heat and Technology, vol. 31, no. 2, pp. 17-26, 2013.

[10] S. E. Rafiee, M. Rahimi and N. Pourmahmoud, "Threedimensional numerical investigation on a commercial vortex tube based on an experimental model- Part I: Optimization of the working tube radius," International Journal of Heat and Technology, vol. 31, no. 1, pp. 49-56, 2013.

[11] S. E. Rafiee and M. M. Sadeghiazad, "3D numerical analysis on the effect of rounding off edge radius on thermal separation inside a vortex tube," International Journal of Heat and Technology, 33(1), pp. 83-90, 2015.

[12] N. Pourmahmoud, A. Hasanzadeh, S. E. Rafiee and M. Rahimi, "Three dimensional numerical investigation of effect of convergent nozzles on the energy separation in a vortex tube," International Journal of Heat and Technology, vol. 30, no. 2, pp. 133-140, 2012.

[13] S. Y. Im and S. S. Yu, "Effects of geometric parameters on the separated air flow temperature of a vortex tube for design optimization," Energy, vol. 37, no. $1, \quad$ pp. 154-160, 2012. DOI: 10.1016/j.energy.2011.09.008.

[14] N. Pourmahmoud, S. E. Rafiee, M. Rahimi and A. Hasanzadeh, "Numerical energy separation analysis on the commercial Ranque-Hilsch vortex tube on basis of application of different gases," Scientia Iranica., vol. 20, no. 5, pp. 1528-1537, 2013.

[15] X. Han, N. Li, K. Wu, Z. Wang, L. Tang, G. Chen and $\mathrm{X}$. Xu, "The influence of working gas characteristics on energy separation of vortex tube," Applied Thermal Engineering, vol. 61, no. 2, pp. 171-177, 2013. DOI: 10.1016/j.applthermaleng.2013.07.027.

[16] S. E. Rafiee and M. M. Sadeghiazad, "Threedimensional numerical investigation of the separation process inside vortex tube using different operating conditions," Journal of Marine Science and Application, First online: 06 April 2016, pp. 1-10, DOI: 10.1007/s11804-016-1348-8.

[17] S. E. Rafiee and M. M. Sadeghiazad, "Effect of conical. valve angle on cold-exit temperature of vortex tube," Journal of Thermophysics and Heat Transfer, vol. 28, pp. 785-794, 2014. DOI: 10.2514/1.T4376.
[18] S. E. Rafiee and M. M. Sadeghiazad, "Threedimensional and experimental investigation on the effect of cone length of throttle valve on thermal performance of a vortex tube using $\mathrm{k}-\varepsilon$ turbulence model," Applied Thermal Engineering, vol. 66, no. s $1-2, \quad$ pp. 65-74, 2014. DOI: 10.1016/j.applthermaleng.2014.01.073.

[19] S. E. Rafiee and M. Rahimi, "Experimental study and three-dimensional (3D) computational fluid dynamics (CFD) analysis on the effect of the convergence ratio, pressure inlet and number of nozzle intake on vortex tube performance-Validation and CFD optimization," Energy, vol. 63, pp. 195-204, 2013. DOI: 10.1016/j.energy.2013.09.060.

[20] S. E. Rafiee, M. M. Sadeghiazad and N. Mostafavinia, "Experimental and numerical investigation on effect of convergent angle and cold orifice diameter on thermal performance of convergent vortex tube," J. Thermal Sci. Eng. Appl, vol. 7, no. 4. DOI: 10.1115/1.4030639.

[21] Y. Xue, M. Arjomandi and R. Kelso, "Energy analysis within a vortex tube," Experimental Thermal and Fluid Science, vol. 52, pp. 139-145, 2013. DOI: 10.1016/j.expthermflusci.2013.09.004.

[22] S. E. Rafiee and M. M. Sadeghiazad, "3D CFD exergy analysis of the performance of a counter flow vortex tube," International Journal of Heat and Technology, vol. 32, no. 1-2, pp. 71-77, 2014.

[23] S. E. Rafiee, and M. Rahimi, "Three-dimensional simulation of fluid flow and energy separation inside a vortex tube," Journal of Thermophysics and Heat Transfer, vol. 28, pp. 87-99, 2014. DOI: 10.2514/1.T4198.

[24] E. Lorenzini and M. Spiga, "Aspetti fluidodinamici della separazione isotopica mediante tubi a vortice di Hilsch Ingegneria," no. 5-6, pp. 121-126, maggiogiugno 1982.

[25] N. Pourmahmoud, M. Rahimi, S. E. Rafiee, and A. Hasanzadeh, "A numerical simulation of the effect of inlet gas temperature on the energy separation in a vortex tube," Journal of Engineering Science and Technology, vol. 9, no. 1, pp. 81-96, 2014. 